 \\ REVISTA INTERNACIONAL DE TRABAJO SOCIAL Y CIENCIAS SOCIALES INTERNATIONAL JOURNAL OF SOCIAL WORK AND SOCIAL SCIENCES
}

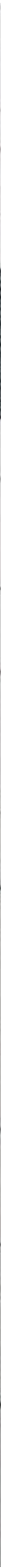

IVAN N. MARTINEZ-SALAZAR | ERIKA ACEVEDO-STEFANONI | SANTIAGO BOIRA | CHAIME MARCUELLO-SERVÓS | LAURA OTERO BELÉN SANZ BARBERO | CARMEN VIVES-CASES | MARÍA JOSÉ RODRÍGUEZ SÁNCHEZ | MATILDE BLANCO VENZALÁ CLARA FERNÁNDEZ BURRACO | ROSER MANZANERA RUIZ | GLORIA ÁLVAREZ-BERNARDO | NURIA ROMO-AVILÉS JOSÉ AZOH BARRY | FRANCISCO JAVIER GORJON GÓMEZ 


\title{
Femicidio y feminicidio: Un análisis de las aportaciones en clave iberoamericana
}

\section{Femicide and Feminicidio: an analysis in Latinoamerican perspective}

Santiago Boira ${ }^{*}$, Chaime Marcuello-Servós ${ }^{* *}$, Laura Otero***, Belén Sanz Barbero**** Y Carmen Vives-Cases ${ }^{* * * *}$

* sboira@unizar.es. GESES, Universidad de Zaragoza

** chaime@unizar.es. GESES, Universidad de Zaragoza

*** laotero@isciii.es. Instituto de Salud Carlos III. CIBER de Epidemiología y Salud Pública. CIBERESP, Madrid, España

**** bsanz@isciii.es. Instituto de Salud Carlos III. CIBER de Epidemiología y Salud Pública. CIBERESP, Madrid, España

***** cvivescases@gmail.com. Grupo de Investigación de Salud Pública de la Universidad de Alicante \& CIBER

de Epidemiología y Salud Pública

\begin{abstract}
Abstrac:
Violence against women is one scourge of our society far of eradication. the death of the victim is the extreme version and irreversible end. From the mid 70s of last century, Diane Russell made visible the phenomena and coined femicide as the murder of a woman being a woman. Later, in the 90s, Lagarde created the neologism feminicidio to translate the work of Russell into Spanish. Since both terms have created a space for knowledge, research and political action. The purpose of this work is to find out how it has addressed feminicidio / femicide in the social sciences in the Latin American context. This has been chosen by a review of the literature in Spanish and Portuguese, analyzing their treatment and the scope of the terms.
\end{abstract}

Keywords: Femicide, femicidio, violence against women.

\section{Resumen:}

La violencia contra las mujeres es una lacra de nuestra sociedad que no se consigue erradicar. En su versión extrema e irreversible terminar con la muerte de la víctima. Desde mediados de la década de los años 70, del siglo pasado y de la mano de Diane Russell se visibilizaron los femicidios como el asesinato de una mujer por el hecho de ser mujer. Posteriormente, en los años 90, Marcela Lagarde crea el neologismo feminicidio al traducir la obra de Rusell al español. Desde entonces ambos términos han creado un espacio de conocimiento, de investigación y de acción política. El propósito de este trabajo es averiguar cómo se ha abordado el femicidio / feminicidio en las ciencias sociales en el contex-

1 Integrantes del equipo español de COST ACTION IS-1206 Femicide Across Europe. 
to iberoamericano. Para ello se ha optado por una revisión de la literatura especializada en español y en portugués, analizando el tratamiento que reciben así como el alcance de los términos.

Palabras clave: Femicidio, feminicidio, violencia contra las mujeres.

\section{Article info:}

Received: 01/02/2015 / Received in revised form: 21/04/2015

Accepted: 25/04/2015 / Published online: 25/06/2015

DOI: http://dx.doi.org/10.5944/comunitania.10.2

\section{Introducción}

La violencia contra las mujeres sigue siendo una de las lacras de nuestra sociedad. Es un asunto trágico que tiene distintas dimensiones. La más visible e irremediable es la que termina con la muerte de la víctima. Es un crimen, un asesinato que hasta hace un tiempo era recogido bajo el concepto de "homicidio"Término que el diccionario del español acota bien como (i). "Muerte causada a una persona por otra" o bien como (ii). "Delito consistente en matar a alguien sin que concurran las circunstancias de alevosía, precio o ensañamiento". Sin embargo, desde mediados de la década de los 70 se ha impulsado un cambio en la visión y denominación del hecho. Fue un cambio consciente y premeditado. Era un giro en la denominación que tenía un claro contenido político, pero también implicaciones epistemológicas y metodológicas. Ese cambio se produce en el mundo anglosajón de la mano de Diane Russell. ${ }^{2}$

Hasta entonces, en inglés también se utilizaba de forma general el término "homicide" - "the deliberate and unlawful killing of one person by another; murder" (Webster Dictionary) - que también tenía un carácter aparentemente técnico pero, desde la perspectiva de Russell, claramente machista. La intención de esta autora fue hacer visible una parte de la realidad que quedaba oculta a los ojos de la sociedad. Quería resaltar aquellos crímenes que eran resultado de la violencia contra las mujeres. Con el término "femicide" quería ir más allá y enfatizaba la muerte de las mujeres por el hecho de ser mujeres, en tanto su condición de género femenino. Como ella ha enunciado más recientemente: "As long ago as 1976, I chose the new term femicide to refer to the killing of females by males because they are female" (Rusell, 2013:19).

2 Sobre este punto veáse http://www.dianarussell.com/origin_of_femicide.html incluye el texto The Origin And Importance OfThe Term Femicide, December, 2011, junto con su Audio y Video. Último acceso 1 de febrero de 2014. 
Así pues, desde que Diane Russell potenciase el uso del término inglés "femicide" en 1976 en el foro del Tribunal on Crimes Against Women, en Bruselas se han elaborado numerosos documentos y trabajos en torno a ese concepto y lo que supone. Cuando Diane Russell presentó en público su propuesta, retomaba una palabra existente, pero lo hacía con un propósito: dar visibilidad a las mujeres y luchar contra la violencia que sufren en numerosos contextos y lugares. Su posición tenía un fuerte contenido "politizante" en una corriente que hay que incluir en el marco de las teorías feministas, pero también en la relación entre violencia, sociedad y estado. Posteriormente otras autoras como Campbell y Runyan (1998) amplian este concepto para incluir en él muertes provocadas por acciones u omisiones que no constituyen un delito, o bien, no pueden ser imputadas a una persona. Así bajo este concepto más amplio se incluirían las muertes de mujeres asociadas a la desnutrición selectiva por razones de género, al aborto inseguro, al infanticidio de mujeres, la trata de mujeres para la prostitución o el tráfico de drogas, a la falta de acceso al sistema sanitario por razones de género, entre otros.

Desde entonces se han producido numerosos avances tanto teóricos como prácticos. Se han incrementado los recursos y las acciones encaminadas a tomar conciencia y erradicar esta lacra. Entre ellos cabe destacar la celebración de encuentros y declaraciones internacionales en distintos contextos como los del International Council of Women ${ }^{3}$ y la Declaración de Viena sobre el Femicidio [Vienna Declaration on Femicide] de 2013. ${ }^{4}$ A pesar de todo, tanto la violencia contra las mujeres como el número de femicidios ha seguido incrementadose. Así lo destacó el Simposium de Viena sobre Femicidio, celebrado en noviembre de 2012 en la sede de Naciones Unidas de Viena. Tras dicho simposium, el consejo Económico y Social de la ONU lanzó en el mes de Abril de 2013, la declaración de Viena sobre Femicidios reconociendo lo alarmante que resulta la violencia contra las mujeres en todo el mundo y dicen: "Alarmados por el hecho de que el feminicidio está aumentando en todo el mundo y, a menudo queda impune, lo cual no sólo intensifica la subordinación y la impotencia de las mujeres y las niñas, sino que también envía el mensaje negativo a la sociedad que la violencia contra las mujeres puede ser a la vez aceptable e inevitable.5 Y concluye con una llamada a la creación de una plataforma donde compar-

\footnotetext{
3 Veáse en: http://www.icw-cif.com/home, último acceso 10 de febrero de 2014.

4 Se puede consultar en: http://www.icw-cif.com/news/22-vienna-declaration-on-femicide, último acceso 12 de marzo de 2014.

5 La traducción es propia. El original dice: Alarmed by the fact that femicide is increasing all over the world and often remains unpunished, which not only intensifies the subordination and powerlessness of women and girls, but also sends the negative message to society that violence against females may be both acceptable and inevitable.

6 El punto 9 dice: Call for the creation of a platform where lawyers, prosecutors, judges, law enforcement officials, academics, feminists, non-governmental organizations, United Nations agencies,
} 
tir conocimientos y buenas prácticas para erradicar esta lacra de todos los países. ${ }^{6}$ Desde que se empezó a utilizar el concepto "femicidio" hasta ahora han transcurrido casi cuatro décadas de acción y concienciación.

En estos años también ha sido clave la traducción de la obra de Russell al español de la mano de Marcela Lagarde - creando el neologismo "feminicidio" - . El concepto de feminicidio trata de incorporar la misoginia que hay en este tipo de asesinatos así como la responsabilidad del Estado al favorecer la impunidad ante estos. A partir de este hecho el estudio del femicidio/feminicidio comienza a adquirir una mayor presencia y relevancia en el mundo de habla hispana y en la producción científica sobre estos temas. Aunque Diane Russell ${ }^{7}$ observa algunas reticencias respecto del concepto de "feminicidio" tal como se ha ido consolidando tras la versión de Lagarde, ambos términos parecen oportunos a los autores para el propósito de este trabajo.

Considerando este marco de referencia, este trabajo pretende responder a la pregunta ¿cómo se ha abordado el femicidio / feminicidio en las ciencias sociales en el contexto iberoamericano? Para ello las y los autores han optado por una revisión de la literatura especializada. Por tanto, el objetivo es analizar el tratamiento que reciben así como el alcance de los términos feminicidio y femicidio en publicaciones científicas y libros de referencia publicados en español y en portugués.

\section{Metodología, proceso y criterios de búsqueda}

Se ha realizado una búsqueda sistemática en las principales bases de datos y repositorios. En concreto, se han revisado las siguientes: REDALYC, DIALNET, REBIUN, SCIELO, SCIENCE DIRECT, ISI WEB OF KNOWLEDGE, PUBMED y LILACS. Se dejan fuera los trabajos de autores hispanohablantes en publicaciones en otras lenguas, especialmente, inglés, por ir más allá del propósito de este trabajo.

Las palabras clave para realizar la búsqueda han sido "feminicidio" y "femicidio". Se ha optado por una búsqueda restringida y dirigida únicamente a estos dos términos por tres motivos. Primero, la inclusión en los criterios de búsqueda de otras palabras clave relacionadas con la violencia y el asesinato de mujeres hubiera aportado resultados más amplios. Sin embargo, para este trabajo en el que se pretende profundizar en los aspectos conceptuales y semánticos en los términos feminici-

governmental and inter-governmental institutions and other relevant actors could share their expertise and good practices, in order to transfer knowledge across regions.

7 Sobre este tema veáse http://www.dianarussell.com/origin_of_femicide.html incluye el textoThe Origin And Importance OfThe Term Femicide, December, 2011, (For a video of Diana presenting this speech, please visit her Audio and Video). Último acceso 20 de diciembre de 2013. 
dio/femicidio, el empleo explícito de alguno de estos dos términos resulta ser un criterio esencial. Segundo, optar por una búsqueda más restringida permite identificar las principales áreas de conocimiento y tópicos que quedan conectadas con estos dos términos. Tercero, este rastreo no persigue realizar una búsqueda sobre aquellas publicaciones que tratan sobre las violencia contra las mujeres sino aquéllas que aunque traten los diferentes tipos de violencia de género conectan en algún punto del texto con el término femicidio o feminicidio.

El procedimiento de trabajo se ha estructurado en los pasos que se describen a continuación. En primer lugar, se realizó una búsqueda general en los repositorios antes nombrados hasta agosto de 2013, donde se obtuvieron un total de 470 registros. Éstos se agruparon creando una primera base de datos.

En segundo lugar, se depuró el conjunto de las referencias obtenidas en función de los siguientes criterios: a) se suprimieron las duplicadas; b) se eliminaron las publicadas en idiomas distintos al español o el portugués; ${ }^{8} \mathrm{c}$ ) se eliminaron las referencias en las que el uso de alguna de las dos palabras clave fue circunstancial, así como aquellas en las que la temática de la publicación se alejaba sensiblemente de los posibles tópicos relacionados con el feminicidio/femicidio y d) se suprimieron los registros referidos a reseñas de libros que en muchos de los casos aparecen de suyo. Después de este proceso de depuración, el número de registros obtenidos quedó reducido a $231 .^{9}$

Se clasificaron los artículos finalmente seleccionados en tres categorías:

- CENTRAL. Se analiza específicamente el asesinato de mujeres (feminicidio/femicidio).

- PRÓXIMA. Se aborda el feminicidio pero asociado a la temática general de la violencia hacia las mujeres.

- COLATERAL. Son trabajos que rozan e incluso nombran el feminicidio / femicidio sin llegar a ser la preocupación nuclear, pero si que hacen alguna referencia a ello.

Adicionalmente, se construyó una base de datos en la que se consideraron las siguientes variables: (i).Tipo de referencia (artículo científico, capítulo del libro, libro completo); (ii). Autores; (iii). Título Primario; (iv). Título de la publicación periódica; (v). Año de publicación; (vi). País de publicación; (v). País como área a la que se refiere al estudio (en el caso de que ésta quede definida bien en el título o en el resumen

\footnotetext{
8 En aquellos casos en los que no ha sido posible encontrar el artículo completo pero sí estaba disponible el abstract en español como en portugués se ha considerado la referencia.

9 No se han considerado los informes de organismos multilaterales como la ONU, OMS, OPS u otro tipo de organizaciones de mujeres, salvo aquellos publicados expresamente en revistas dentro de los repositorios reseñados.
} 
del trabajo). (vi). Resumen. Como complemento a revisión de la búsqueda se utilizó la herramienta Refworks. La elección de las variables ha estado condicionada a las posibilidades que ofrece esta herramienta que es versátil pero con limitaciones.

Por último, se realizó un análisis de contenido de los resúmenes de aquellos que estaban disponibles y accesibles de manera telemática. Cuestión que no fue posible en todos los casos por la dificultad para su localización.

\section{Relevancia, caracterización y "geografía" de las publicaciones identificadas}

De las 231 referencias de la base depurada, 81 registros han sido asignados a la Categoría Central, 81 a la Categoría Próxima y 69 a la Categoría Colateral. De las referencias encontradas 213 corresponden a artículos en revistas científicas y 18 a libros, capítulos de libro o monografías.

Respecto a la fecha de estas publicaciones, no se ha encontrado ninguna referencia con anterioridad al año 2000, siendo el año 2012, el año que concentra el mayor número de trabajos publicados, 44 lo que representa el 19\% del total. Resulta también significativo el salto en el número de publicaciones a partir del año 2005. En la tabla I se muestra la evolución anual del total de trabajos.

TABLA I. Evolución de las publicaciones

\begin{tabular}{|ccc|}
\hline Año & $\mathbf{N}$ & $\%$ \\
\hline 2000 & 3 & 1,3 \\
\hline 2001 & 1 & 0,4 \\
\hline 2002 & 5 & 2,2 \\
\hline 2003 & 3 & 1,3 \\
\hline 2004 & 2 & 0,9 \\
\hline 2005 & 8 & 3,5 \\
\hline 2006 & 20 & 8,7 \\
\hline 2007 & 23 & 10,0 \\
\hline 2008 & 23 & 10,0 \\
\hline 2009 & 25 & 10,8 \\
\hline 2010 & 34 & 14,7 \\
\hline 2011 & 29 & 12,6 \\
\hline 2012 & 44 & 19,0 \\
\hline 2013 & 11 & 4,8 \\
\hline Total & $\mathbf{2 3 1}$ & $\mathbf{1 0 0 , 0}$ \\
\hline
\end{tabular}


De hecho, es a partir del año 2000 cuando aparecen los primeros estudios que sitúan el foco en México y hacen visibles los asesinatos en Ciudad Juárez. Monárrez (2000; $2002 ; 2009$ ) aborda decididamente este problema a partir del la traducción de Marcela Lagarde del marco planteado por Radford y Rusell (1992) y Rusell (2006) en el que el feminicidio se entiende como una consecuencia de la inequidad y de las relaciones de poder entre hombres y mujeres que justifica y consiente el abuso de los cuerpos de las niñas y de las mujeres, y su asesinato. Entre los objetivos que se plantean se encuentran los siguientes: (i). definir las distintas clases de feminicidios, (ii). identificar un perfil sociodemografico de la víctima y (iii). clasificar la relación de parentesco entre la víctima y el agresor. Asímismo, Lagarde $(2006 ; 2009)$ profundiza en el debate conceptual estableciendo diferencias entre las categorías de femicidio y feminicidio.

Este asunto marca sin duda el campo semántico y conceptual desde donde se va a desarrollar el concepto de feminicidio y la "geografía" donde se realizan los principales estudios en el ámbito latinoamericano. Además, es también determinante en cuanto al posicionamiento de algunas de las principales autoras respecto al debate entre el par femicidio/feminicidio. Si se atiende a este aspecto en las referencias encontradas en los artículos de investigación (Categoría Central y Próxima) donde se hace referencia a algún país en concreto - lo que ocurre en 124 de los 160 trabajosse observa que en 48 de ellos (el 38,7\%) el país referido es México. Además, otro dato importante a reseñar es que para el caso de México una parte mayoritaria de estos trabajos se refieren a Ciudad Juárez. En relación a los libros, capítulos de libro o monografías publicadas es similar. De las 18 referencias encontradas 10 de ellas se refieren a México. En el Tabla II se muestra la relación de artículos por país.

TABLA II. Distribución “geográfica" de las publicaciones

\begin{tabular}{|ccc|}
\hline País & $\mathbf{N}$ & $\%$ \\
\hline Argentina & 6 & 4,8 \\
\hline Brasil & 8 & 6,5 \\
\hline Chile & 9 & 7,3 \\
\hline Colombia & 11 & 8,9 \\
\hline Costa Rica & 5 & 4,0 \\
\hline Cuba & 1 & 0,8 \\
\hline España & 17 & 13,7 \\
\hline Guatemala & 4 & 3,2 \\
\hline México & 48 & 38,7 \\
\hline Panamá & 2 & 1,6 \\
\hline Perú & 6 & 4,8 \\
\hline Puerto Rico & 2 & 1,6 \\
\hline República Dominicana & 3 & 2,4 \\
\hline Venezuela & 2 & 1,6 \\
\hline Total & $\mathbf{1 2 4}$ & $\mathbf{1 0 0 , 0}$ \\
\hline
\end{tabular}


Otro aspecto significativo es que, con excepción de España y Portugal, no se han encontrado ni en lengua española ni portuguesa trabajos que hagan referencia ni a otros países del resto de Europa ni de otras regiones del mundo. Pese a que en algunas de las publicaciones se pone de relieve el hecho de que el fenómeno del feminicidio de Ciudad Juárez entendido como "zona 0" debe servir como referencia y motor que visibilice los asesinatos de mujeres por razones de género en otras partes de Latinoamérica, se observa que las publicaciones en español y portugués que abordan este problema en otros contextos son limitadas.

En algunos casos, sobre todo de ámbito legal, se estudian los pasos que se van dando de cara a adaptar las legislaciones o a definir el feminicidio como tipo legal específico. Así, en algunos trabajos (Ginés, 2010; Jiménez, 2011, Mujica y Tuesta, 2013; Munevar, 2012) se abordan los aspectos legales del feminicidio, su tipificación, su conexión con los derechos humanos y el estado de la legislación para el caso de países concretos. Por otra parte, en el conjunto global de las publicaciones aparecen pocos estudios que traten de concretar la prevalencia del fenómeno, y los que hay están fundamentalmente centrados en México (Monárrez, 2000; Fuentes y González, 2008), España (Fernández, 2011; Vives-Cases et al., 2008), Argentina (Fernández, 2010) y Costa Rica (Carcedo y Sagot, 2002). Asimismo, en alguno de los trabajos se analiza el femicidio en relación con alguna circunstancia específica, por ejemplo Crempien (2007) analiza el impacto de la violencia de género y el femicidio en mujeres embarazadas. Respecto a los estudios de prevalencia, algunos autores se preguntan por el modo con el cual las estadísticas tratan este tipo de violencia y la posible tendencia al ocultamiento. Así por ejemplo, en Fernández (2012) se plantean para el caso de Argentina cómo algunos casos de suicidios de mujeres pueden estar encubriendo casos de asesinato. Pronto también se cae en la cuenta de la dificultad para establecer una estadística fiable de las mujeres muertas debido a las connotaciones ideológicas y políticas de este problema (Pérez y Padilla, 2002).

\section{Sobre las Revistas}

Para el análisis de este apartado sólo se han manejado las revistas de la Categoría Central y Próxima donde se han publicado las referencias estudiadas directamente relacionadas con la temática del femicidio/feminicidio y la violencia contra la mujer ( $\mathrm{N}=160$ ). Para el análisis de dichas revistas científicas cabe preguntarse por su procedencia, calidad y relevancia así como por sus áreas de especialización, puesto que pueden aportar algunos aspectos interesantes sobre el modo y los contextos en los que es tratado el femicidio/feminicidio.

Respecto a la procedencia de las revistas que han publicado las referencias nuevamente México aparece como el país que más publicaciones acoge $(N=41)$, seguido de España, Colombia y Brasil. El conjunto de estos cuatro paises suponen el $71 \%$ de todas las publicaciones. Los resultados se muestran en la tabla III. 
TABLA III. País de edición de las revistas

\begin{tabular}{|ccc|}
\hline País & N & $\%$ \\
\hline México & 41 & 28,3 \\
\hline España & 36 & 24,8 \\
\hline Colombia & 15 & 10,3 \\
\hline Brasil & 11 & 7,6 \\
\hline Chile & 9 & 6,2 \\
\hline Costa Rica & 7 & 4,8 \\
\hline Perú & 6 & 4,1 \\
\hline Venezuela & 5 & 3,4 \\
\hline República Dominicana & 3 & 2,1 \\
\hline USA & 3 & 2,1 \\
\hline Argentina & 3 & 2,1 \\
\hline Portugal & 2 & 1,4 \\
\hline Puerto Rico & 2 & 1,4 \\
\hline Ecuador & 1 & 0,7 \\
\hline Suiza & 1 & 0,7 \\
\hline Total & $\mathbf{1 4 5}$ & $\mathbf{1 0 0}, \mathbf{0}$ \\
\hline
\end{tabular}

Otro aspecto a considerar es la calidad de las revistas en las que aparecen estos trabajos. De las 142 revistas, únicamente 17 se encuentran indexadas en la ISI Web of Knowledge. Por otra parte, si se considera la Clasificación Integrada de Revistas Científicas (CIRC) los resultados que se obtienen en las diferentes categorías se muestran en la tabla IV.

\section{TABLA IV. Clasificación Integrada de Revistas Científicas (CIRC)}

\begin{tabular}{|lcc|}
\hline CIRC & N & $\%$ \\
\hline A $($ SI) & 17 & 12,0 \\
\hline B & 15 & 10,6 \\
\hline C & 32 & 22,5 \\
\hline D & 19 & 13,4 \\
\hline Sin Incluir & 59 & 41,5 \\
\hline Total & $\mathbf{1 4 2}$ & $\mathbf{1 0 0}$ \\
\hline
\end{tabular}

Hay que recordar que en esta clasificación en el grupo A se incluyen las revistas científicas de mayor nivel. Pertenecerían al mismo las revistas internacionales de más prestigio que han superado procesos de evaluación muy exigentes para el ingreso en diferentes bases de datos. El grupo B está compuesto por revistas cientí- 
ficas españolas de calidad pero que no alcanzan un alto nivel de internacionalización, aunque son revistas que reciben cierto grado de citación y que respetan los estándares de publicación. Asimismo forman parte de este grupo aquellas revistas científicas internacionales con un menor pero aceptable grado de prestigio y difusión. El grupo C está compuesto por las revistas científicas españolas de segundo orden que, o bien son poco citadas, o bien no cumplen con los estándares de publicación científica. También se incluyen las revistas internacionales de menor relevancia. Finalmente, el grupo D estaría conformado por todas aquellas publicaciones no incluidas en ninguna de las categorías anteriores $y$, por tanto, con un status científico distinto del marco hegemónico. ${ }^{10}$

Según los datos disponibles, en la tabla IV se muestra que el $41,5 \%$ de las revistas no están incluidas en esta clasificación. Este hecho se ha podido producir por diversas razones y no siempre necesariamente por falta de calidad, este es el caso de alguna revista de carácter biomédico. En cualquier caso, parece claro que un porcentaje significativo de los artículos publicados en español o portugués en esta materia no se ha producido en publicaciones que midan su prestigio en función del modelo dominante basado en el Journal Citation Report de la empresa Thompson. En la tabla $V$ se muestra el listado de las revistas pertenecientes a la Categoría Central indicando el país donde se editan, la clasificación CIRC y si se encuentran indexadas en la Web of Knowledge.

${ }^{10}$ En este caso, cabe plantear la crítica lanzada desde distintos autores y disciplinas. Sirva como ejemplo el caso de Randy Schekman biólogo estadounidense, ganador del Premio Nobel de Medicina en 2013. O basta recordar la conocida Ley de Goodhart que advierte que "when a measure becomes a target, it ceases to be a good measure". 


\section{TABLA V. Revistas Categoría Central. Clasificación CIRC e indexación ISI}

\begin{tabular}{|c|c|c|c|}
\hline Revista & $\begin{array}{l}\text { País } \\
\text { edición }\end{array}$ & $\begin{array}{l}\text { Clasifica- } \\
\text { ción CIRC }\end{array}$ & $\begin{array}{l}\text { Web of } \\
\text { Knowledge }\end{array}$ \\
\hline Ámbitos: Revista internacional de comunicación & España & $\mathrm{C}$ & \\
\hline Desacatos & México & $\mathrm{D}$ & \\
\hline A Trabe de ouro: publicación galega de pensamento crítico & España & & \\
\hline Acta Bioethica & Chile & A & $\mathrm{SI}$ \\
\hline Acta Colombiana de Psicología & Colombia & D & \\
\hline American University International Law Review & USA & & \\
\hline Andamios. Revista de Investigación Social & México & $A$ & $\mathrm{SI}$ \\
\hline Cambio 16 & España & & \\
\hline Colorado Review of Hispanic Studies & USA & & \\
\hline Criminalidad & Colombia & $\mathrm{D}$ & \\
\hline Debate Feminista & México & & \\
\hline Desde el jardín de Freud: revista de psicoanálisis & Colombia & $\mathrm{C}$ & \\
\hline \multicolumn{4}{|l|}{ El Cotidiano } \\
\hline Estudios Sobre el Mensaje Periodístico & España & A & $\mathrm{SI}$ \\
\hline $\begin{array}{l}\text { F@ro: revista teórica del Departamento de Ciencias de la } \\
\text { Comunicación }\end{array}$ & Chile & C & \\
\hline Fermentum. Revista Venezolana de Sociología y Antropología & Venezuela & & \\
\hline Frontera Norte & México & C & \\
\hline Gaceta Sanitaria & España & A & $\mathrm{Si}$ \\
\hline ICEV.Revista d'Estudis de la Violencia & España & D & \\
\hline Iconos. Revista de Ciencias Sociales & Ecuador & C & \\
\hline Interface - Comunicação, Saúde, Educação & Brasil & & \\
\hline Journal of Latin American \& Caribbean Anthropology & USA & $\mathrm{D}$ & \\
\hline Kavilando & Colombia & $\mathrm{D}$ & \\
\hline La Aljaba, Segunda Epoca.Revista de Estudios de la Mujer & Argentina & & \\
\hline Medicina Legal de Costa Rica & Costa Rica & & \\
\hline Nóesis. Revista de Ciencias Sociales y Humanidades & México & & \\
\hline Nomadías & Chile & & \\
\hline Papeles de Población & México & A & $\mathrm{SI}$ \\
\hline Prosopopeya: revista de crítica contemporánea & España & $\mathrm{B}$ & \\
\hline Relaciones internacionales & España & B & \\
\hline Revista Anthropologica & Peru & & \\
\hline Revista Argentina de Salud Pública & Argentina & & \\
\hline Revista Chilena de Derecho & Chile & C & \\
\hline Revista chilena de neuro-psiquiatría & Chile & & \\
\hline Revista Criminalidad & Colombia & D & \\
\hline Revista de Derecho Penal & España & B & \\
\hline Revista de Derecho Penal y Cr iminología & España & B & \\
\hline Revista de Estudios de Género. la ventana & México & & \\
\hline Revista de Saúde Pública & Brasil & & $\mathrm{Si}$ \\
\hline Revista Española de Investigación Criminológica (REIC) & España & B & \\
\hline Revista Española de Salud Pública & España & & SI \\
\hline Revista Estudios Socio Jurídicos & Colombia & C & \\
\hline Revista Estudos Feministas & Brasil & & \\
\hline Revista Europea de Estudios Latinoamericanos y del Caribe & España & $\mathrm{B}$ & \\
\hline Revista logos ciencia y tecnología & Colombia & & \\
\hline Revista Mexicana de Sociología & México & C & \\
\hline Revista Pueblos y fronteras Digital & México & C & \\
\hline Revista Venezolana de Estudios de La Mujer & Venezuela & $\mathrm{D}$ & \\
\hline Sortuz: Oñati Journal of Emergent Socio-legal Studies & España & $\mathrm{C}$ & \\
\hline Tiempo de paz & España & C & \\
\hline Tradução \& Comunicação & Brasil & & \\
\hline Universitas Humanística & Colombia & C & \\
\hline Versants: Revue Suisse des Littératures Romanes & Suiza & & \\
\hline
\end{tabular}


Finalmente, respecto a las materias en las que las revistas quedan incluidas (aqueIlas en las que aparece, tomado del repositorio de DIALNET) el $71 \%$ de las revistas pertenecen al campo de las ciencias sociales o jurídicas. Los resultados se muestran en la tabla VI.

TABLA VI. Agrupación de las revistas por materias (DIALNET)

\begin{tabular}{|lcc|}
\hline Materias & $N$ & $\%$ \\
\hline Ciencias Sociales & 39 & 51,3 \\
\hline Antropología. Etnología & 2 & \\
Generalidades & 16 & \\
Información. Documentación & 3 & \\
Política & 4 & \\
Sociología. Población. Trabajo Social & 14 & \\
Ciencias Jurídicas & 15 & 19,7 \\
\hline Derecho Procesal y Penal & 7 & \\
Derecho Social & 1 & \\
Generalidades & 6 & \\
Dcho Internacional & 1 & \\
Psicología y Educación & 12 & 15,8 \\
\hline Generalidades & 1 & \\
Psicología & 11 & \\
Humanidades & 5 & 6,6 \\
\hline Generalidades & 4 & \\
Filosofía. Ética & 1 & \\
Filologías & 2 & 2,6 \\
\hline Historia y Crítica Literaria & 2 & \\
Ciencias de la Salud & 2 & 2,6 \\
\hline Generalidades & 1 & \\
Psiquiatría & 1 & \\
Economía y Empresa & 1 & 1,3 \\
\hline Economía Aplicada & 1 & \\
\hline
\end{tabular}

\section{Femicidio y feminicidio: un análisis de contenido}

Si no se hacen distingos entre el término femicidio tal como lo plantea Diana Russell y la traducción de feminicidio de Lagarde, entonces, tal y como apunta Laurenzo (2012) la categoría feminicidio ha sido promovida por la teoría feminista no sólo para visibilizar las causas verdaderas por las que muchas mujeres mueren sino también para impulsar cambios tanto políticos, legislativos, como sociales en diferentes países iberoamericanos incorporando esta figura. 
Los feminicidios aparecen asociados a algunos temas clave como son los llamados "crímenes pasionales" amparados en muchos países por la justicia como ocurre para el caso colombiano (Castillo, 2009). También en diferentes trabajos se alude al femicidio como un problema de salud pública (Álvarez, 2003). Por otra parte, el feminicidio entendido como la "forma más extrema del terrorismo sexista" (Fernández, 2012). Finalmente cabe destacar tambien la relación entre feminicidios y violación sexual, claramente identificada en un contexto extremo como Ciudad Juárez que Monárrez (2009) define como "feminicidio sexual sistémico".

Según queda claro en muchos de los trabajos revisados, el feminicidio constituye una vulneración grave de los derechos humanos. Esta asociación aparece de manera permanente en muchas de las referencias, lo cual sugiere que no se pueden contemplar el problema desde una posición individual o que pudiera afectar únicamente al ámbito privado o familiar. Desde este punto de vista, aparecen diferentes escenarios y teorías que otros sujetos sociales involucrados en el hecho violento ofrecen para explicar y/o justificar los asesinatos. Así, por ejemplo, para el caso de escenarios bélicos como en Colombia, el asesinato de las mujeres puede quedar invisibilizado o reducido a anécdota por el propio contexto de guerra. Del mismo modo sucede en las constantes referencias a mafias, la denominada "narco-cultura", las estructuras corruptas de algunos estados, la impunidad, o las condiciones de pobreza que pueden estar detrás de los femicidios. Todos estos elementos constituyen elementos esenciales del propio sistema dinámico que pueda ayudar a descifrar este tipo de violencia. Esta visión del problema que pretende ser sistémica y holística queda también apuntada en algunas de las referencias que componen esta revisión.

Asimismo, en algunos trabajos se abordan diferentes lados del problema que pueden reforzar la visión holística y sistémica que se propone. Así, por ejemplo, Domínguez y Ravelo (2003:122) tratan de analizar "las diversas hipótesis propuestas por los enunciadores sociales, entre los que se incluyen a las instituciones de los tres niveles de gobierno, la prensa, el medio académico, las Organizaciones No Gubernamentales (ONG), los partidos políticos y las instituciones religiosas." Estos discursos se consideran como actos de habla, traspasando el criterio de verdad o falsedad. Lo que los autores quieren destacar son las condiciones de enunciación, la perspectiva teórica o ideológica que las sustenta, y su uso como acto político. Este estudio del discurso social de la violencia contra mujeres trata de destacar la manera en que se instrumentaliza la victimización en el campo de las relaciones de poder del contexto fronterizo".

Pérez (2005) analiza el desarrollo de las ONG de Ciudad Juárez explorando la acción colectiva femenina revelando las convergencias y divergencias así como sus relaciones con las madres de las víctimas y el gobierno. Freyermuth y Argüello (2010:181) aportan una visión en la que se acentúan las consecuencias de una violencia estructural traducida en un elevado número de muertes prematuras de mujeres en el territorio mexicano de los Altos de Chiapas. El análisis se centra en las 
"relaciones sociales en el ámbito institucional, entre las personas, y entre las instituciones y las personas" en la que aspectos como la "pobreza, rezago, insensibilidad, naturalización, encubrimiento, omisión o comisión de la violencia", inciden directamente en las condiciones educativas y sanitarias y en las posibilidades de supervivencia.

En relación con este tema, algunas investigaciones (Godoy-Paiz, 2012) confrontan las visiones y los discursos oficiales sobre el feminicidio con las experiencias de las familias de las víctimas poniendo en entredicho algunas de estas visiones institucionales que pueden ser tendentes a minimizar el problema y a convertirlo en algo aislado y privado. Desde la teoría sociológica, Pineda y Herrera (2007: 419) analizan el fenómeno del feminicidio en Ciudad Juárez en relación con el enfoque de la sociedad del riesgo que, como apuntan los autores, "al final se traducirá en una cultura del miedo, la cual supone la rendición individual y colectiva". Se analizan los entramados sociales, las interacciones y dependencias de los diferentes actores así como las complicidades y el silencio de las élites y de la clase política.

También en cuanto a los medios de comunicación las relaciones entre información e impunidad (Gewecke, 2011) o el trabajo de García y Gallur (2012) en el que analizan en qué medida se conforman los valores de la noticia en un contexto de conflicto, con especial referencia a la información sobre el feminicidio de Ciudad Juárez. También en este misma línea Lagos-Lira (2008) analiza en el contexto chileno el modo en que la prensa refuerza los estereotipos y desigualdades de género a partir de la cobertura de las noticias sobre feminicidio. En España, Rodríguez (2008), repasa el tratamiento informativo que se realiza de los asesinatos de mujeres en la redacción periodística analizando la evolución de los términos utilizados o las expresiones más frecuentes para referirse a este tema.

Un aspecto que resulta interesante destacar es el abordaje mutidisciplinar e interdisciplinar de este fenómeno: la criminología, la sociología, la psicología, la antropología, la economía, la salud pública entre otras disciplinas, han abordado esta problemática, si bien también la literatura (Bencomo, 2011) y el cine (Corral, 2011; Hind, 2010).

Considerando los elementos expuestos, las reflexiones planteadas en la literatura revisada presentan dos planos diferenciados. El primero de ellos se relaciona con los substratos teóricos y explicativos donde situar el fenómeno. En este nivel, las creencias distorsionadas de género, las relaciones de poder y la cultura patriarcal se utilizan como elementos explicativos centrales. Sin embargo, junto con esta condición necesaria e imprescindible, se muestra la necesidad de acción u omisión de un Estado que de una u otra manera permita los asesinatos y los deje impunes. Asimismo, dentro también de este plano teórico explicativo, muchas de las referencias conectan y relacionan los feminicidios con los distintos actores sociales coadyuvando con una visión sistémica del problema. En la figura 1 se representan algunas de estas conexiones. 


\section{FIGURA 1. Aspectos teóricos y sustratos explicativos}

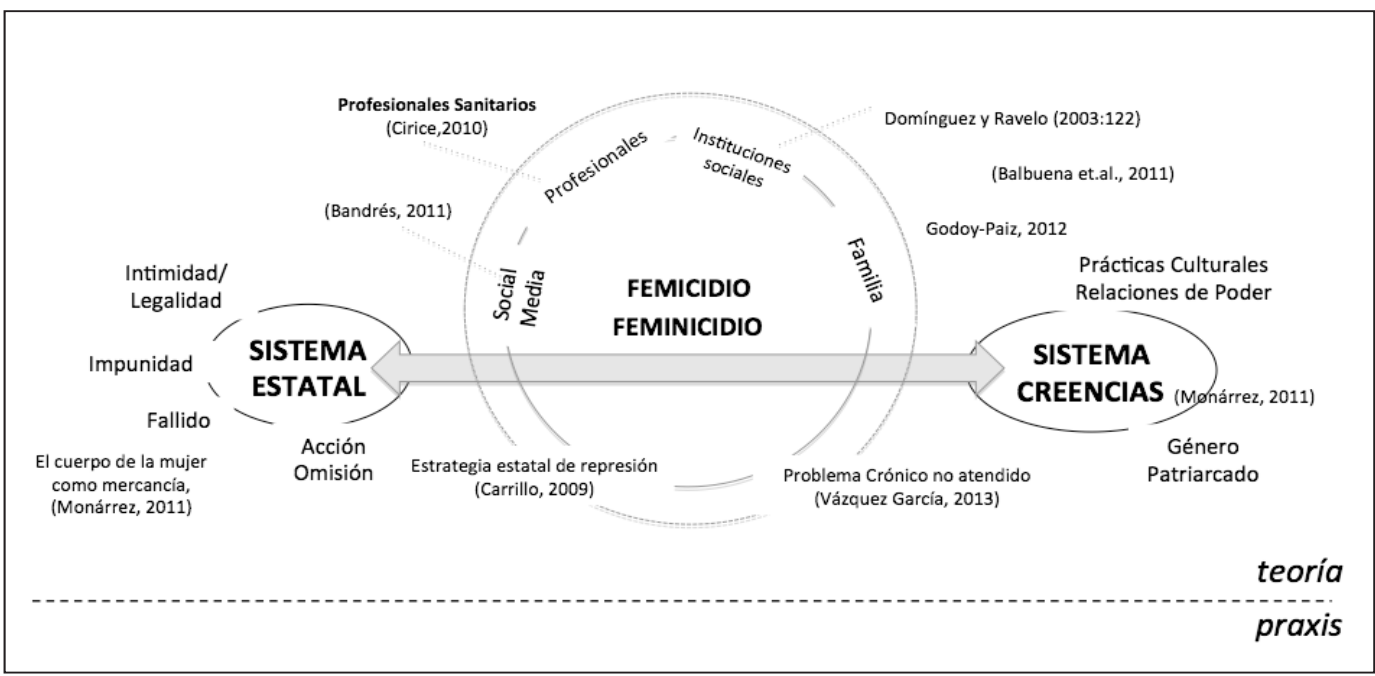

FiguRA 2. Preguntas y acciones

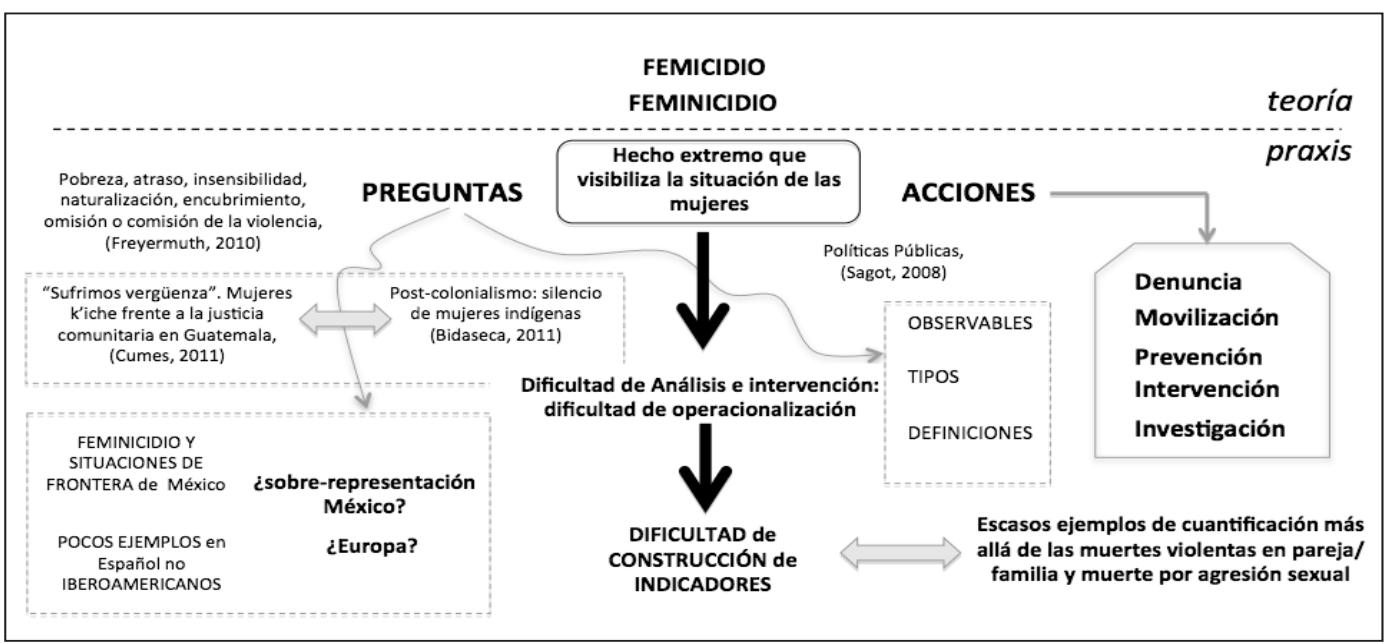

El segundo de los planos de análisis se refiere a las preguntas que el feminicidio suscita y a las posibles acciones para su erradicación. En este sentido cabe preguntarse por cuestiones como el posible impacto y prevalencia de este fenómeno en otras zonas de lberoamérica, por las similitudes o diferencias en los diferentes contextos geográficos y culturales o por el tipo de actuaciones más adecuadas. En este 
último punto hay que destacar la dificultad que parece existir para operacionalizar el fenómeno y la construcción de indicadores. En la figura 2 se muestran algunas de estas cuestiones y las posibles vías de acción sobre le fenómeno.

\section{México y la especificidad de Ciudad Juárez}

El Estado, la guerra y las consecuencias de una ideología patriarcal internalizada aparecen como tres importantes razones que alimentan el binomio asesinato/impunidad. A partir del análisis realizado de los textos, este binomio parece desempeñar un importante papel. Respecto a los feminicidios en el Estado de México, Arteaga y Valdés (2010: 5) consideran que responde a procesos de desafiliación social configurándose como un modo de respuesta "al resquebrajamiento del modelo hegemónico de feminidad y masculinidad". En lo que se refiere a Ciudad Juárez, Monárrez (2012: 197) afirma contundente que: "los dos elementos fundantes de un Estado nación se encuentran colapsados. El territorio es un campo de batalla controlado por el crimen organizado y la delincuencia común; el poder político se encuentra desarticulado y los discursos de la élite política nacional, estatal y municipal dejan en claro que la muerte continuará".

Otras perspectivas analizan el fenómeno de la violencia en la sociedad mexicana como un asunto complejo y multifacético, una de cuyas manifestaciones más destacadas es la violencia feminicida en Ciudad Juárez. Bencomo (2011:13) relaciona el fenómeno violento con "el agotamiento de un Estado de derecho, la crisis del sistema judicial y el aparato político mexicano, la narcocultura y los ritos primitivos que retornan y se potencian en medio de las asimetrías propias del México postmoderno". Algunos autores (Álvarez, 2003) consideran que los feminicidios de Ciudad Juárez no representan casos típicos equiparables a otras zonas geográficas. En primer lugar, aparece con potencia del término "desaparición". Además, se señala que los cadáveres presentan a menudo signos de violación y mutilaciones entre otros. Méndez (2010) y Ravelo y Sánchez (2006) relacionan la violencia en Ciudad Juárez con el territorio maquilador. En este contexto, Sánchez (2007) expone algunos factores que favorecen la violencia de género como las migraciones masivas del campo a la ciudad con el objetivo de obtener un puesto de trabajo, la nómina generalizada o la incorporación de la mujer al mercado laboral.

Por otra parte, Aikin (2012) se adentra en el caso de Ciudad Juárez para analizar cómo ha llegado a convertirse en paradigmático involucrando a activistas, agencias internacionales y gobiernos, para lo que aplican el modelo en espiral de Risse y Sikkink (1999).

Ante esta íntima relación entre feminicio y Ciudad Juárez es posible preguntarse hasta qué punto se trata de una realidad local en la que de una manera drámática parecen coincidir diferentes circustancias que la han hecho posible o por el contra- 
rio es posible entenderla de una manera global en la que Ciudad Juárez ha ayudado a visibilizar y explicitar un fenómeno, "la muerte de mujeres", fenómeno que se extiende mucho más allá del territorio norte de México. Probablemente, en este cáso sería importante identificar los elementos característicos y diferenciadores de las muertes de mujeres en Ciudad Juárez de las producidas en el resto de México y en otras partes del mundo. En la figura 3 aparecen algunas de las relaciones identificadas en este punto.

\section{FiguRA 3. El paradigma de Ciudad Juárez}

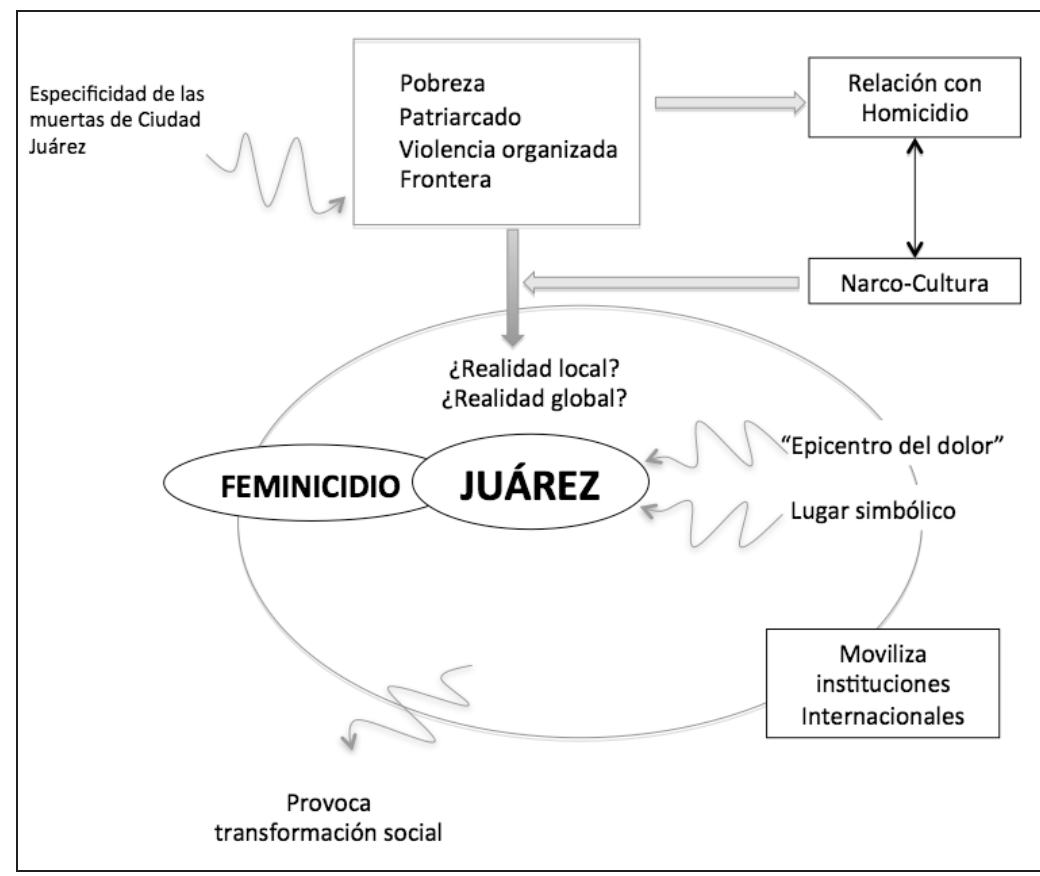

\section{Para seguir}

Tal como señalan (Laurent et al., 2013:1), "femicide is the ultimate form of violence against women and girls and takes multiple forms. Its many causes are rooted in the historically unequal power relations between men and women and in systemic gender-based discrimination. For a case to be considered femicide there must be an implied intention to carry out the murder and a demonstrated connection between the crime and the female gender of the victim. So far, data on femicide have been highly unreliable and the estimated numbers of women who have been victims of femicides vary accordingly. Femicides take place in every country of the world. The 
greatest concern related to femicide is that these murders continue to be accepted, tolerated or justified - with impunity as the norm. To end femicide we need to end impunity, bring perpetrators to justice, and every individual has to change his/her attitude towards women."

En las páginas anteriores se han recogido y analizado los principales resultados de la revisión de la literatura especializada sobre este fenómeno. El cuál sigue siendo un reto para todas la humanidad. Como indica el título Femicide. A Global Issue That Demands Action de la obra colectiva publicada en el año 2013 por el Academic Council on the United Nations System (ACUNS) Vienna Liaison Office, coordinada por Claire Laurent, Michael Platzer and Maria Idomir estamos ante un asunto global, planetario que demanda acciones y decisiones para su erradicación.

El reto es seguir avanzando para erradicar las muertes violentas y mejorar tanto los diagnósticos como la prevención de las mismas.

\section{Referencias}

Aikin, O. 2012. "La agenda del «feminicidio» durante la administración de Vicente Fox: Un estudio de caso en torno a la presión política transnacional". Relaciones Internacionales: Revista académica cuatrimestral de publicación electrónica, 19: 27-55.

Álvarez, J. A. 2003. "Las muertas de Juárez. bioética, género, poder e injusticia". Acta bioethica, 9(2): 219-228.

Arteaga, N. y Valdés, J. 2010. "Contextos socioculturales de los feminicidios en el estado de méxico: Nuevas subjetividades femeninas". Revista mexicana de sociología, 72(1): 5-35.

Bencomo, A. 2011. "Los relatos de la violencia en Sergio González Rodríguez: Huesos en el desierto, el vuelo y el hombre sin cabeza". Andamios, 8(15): 13-35.

Campbell, J. y Runyan, C. W. (1998). "Femicide." Homicide Studies, 2(4): 347-352.

Carcedo, A. y Sagot, M. 2002. "Femicidio en Costa Rica: balance mortal". Medicina Legal de Costa Rica, 19(1): 5-16.

Castillo, N. C. 2009. "¿Por qué razones distintas a la filiación política nos matábamos los colombianos en los años 50?". Universitas humanística, 67: 147-164.

Corral, S. 2011. "Ciudad juárez: La imagen del feminicidio en el cine." En: M. E. Quinteiro (Eds.), La Mujer en la Historia, el Arte y el Cine. Discurso de Género, Variantes de contenido y soportes. De la palabra al Audio-Visual. Salamanca: Ediciones Universidad de Salamanca.

Crempien, C. 2007. "Violencia doméstica durante el embarazo: magnitud del problema y consecuencias". Revista de Psiquiatría Clínica, 44(1): 31-40.

Domínguez, H. y Ravelo, P. 2003. "La batalla de las cruces los crímenes contra mujeres en la frontera y sus intérpretes". Desacatos, 13, 122-133.

Fernández, J. G. 2011. "Feminicidios de género: Evolución real del fenómeno, el suicidio del agresor y la incidencia del tratamiento mediático." Revista Española de Investigación Criminológica (REIC), 9: 1-27. 
Fernández, A. M. 2010. “Estudio cuali-cuantitativo de la mortalidad femenina por causas externas y su relación con la violencia de género". Revista Argentina de Salud Pública, 1(3): 18-23.

Fernández, A. M. 2012. "Femicidios: La ferocidad del patriarcado". Nomadías, 16: 47-73. Freyermuth, G. y Argüello, H. E. 2010. "La muerte prematura de mujeres en Los Altos de Chiapas. Un análisis desde la violencia". Revista pueblos y fronteras digital, 6(10): 181-216.

Fuentes, D. F. y González, I. A. 2008. “Muertes por violencia en las mujeres de Tijuana, Baja California, 1999-2005". Papeles de Población, 14(56): 257-282.

García, B. y Gallur, S. 2012. "La conformación de valores noticia en un contexto de conflicto. Análisis pragmático de su funcionamiento en la información sobre el narcotráfico en México y el feminicidio de Ciudad Juárez". Estudios sobre el mensaje periodistico, 18(2): 491-511.

Gewecke, F. 2010. “Corrupción, impunidad, poder salvaje, masculinidad a ultranza y violencia sin límite: Los feminicidios de ciudad juárez y la narcoviolencia en méxico: Entrevista con Sergio González Rodríguez". Versants: Revue Suisse Des Littératures Romanes/Rivista Svizzera Di Letterature Romanze/Schweizerische Zeitschrift Für Romanische Literaturen, 57(3): 69-78.

Ginés, E. 2010. “El feminicidio de Ciudad Juárez ante la Corte Interamericana de Derechos Humanos". Tiempo de paz, 96: 58-68.

Godoy-Paiz, P. 2012. "No es sólo "otra mujer»: Femicidio y Representación en Guatemala". Journal of Latin American \& Caribbean Anthropology, 17(1): 88-109.

Hind, E. 2010. “Estado de excepción y feminicidio: El traspatio/Backyard (2009) de Carlos Carrera y Sabina Berman". Colorado Review of Hispanic Studies, 8: 27-42.

Jiménez, N. P. 2011. "Femicidio/Feminicidio: una salida emergente de las mujeres frente a la violencia ejercida en contra de ellas". Revista logos ciencia y tecnología, 5: 127-148.

Lagarde, M. 2006. "Del femicidio al feminicidio". Desde el jardín de Freud: revista de psicoanálisis, 6: 216-225.

Lagarde, M. 2009. "Claves feministas en torno al feminicidio". En: E. Molina y N. San Miguel (Coords.), Nuevas líneas de investigación en género y desarrollo. Madrid: Universidad Autónoma de Madrid.

Lagos-Lira, C. 2008. “Una tipología del feminicidio según la prensa chilena: Manifestación de la violencia de género". F@ro: Revista Teórica del Departamento de Ciencias de la Comunicación, 8.

Laurenzo, P. 2012. "Hace falta un delito de feminicidio". Revista de Derecho Penal, 20: 243-256.

Laurent, C., Platzer, M. y Idomir, M. 2013, Femicide A Global Issue that Demands Action. Vienna: Academic Council on the United Nations System (ACUNS) Vienna Liaison Office.

Méndez, L. H. 2010. "Territorio maquilador y violencia. el caso de Ciudad Juárez". El Cotidiano, 164: 27-40.

Mujica, J.. y Tuesta, D. 2013. "Problemas de construcción de indicadores criminológicos y situación comparada del feminicidio en el Perú". Anthropologica, 30: 169-194.

Monárrez, J. E. 2000. “La cultura del feminicidio en Ciudad Juárez, 1993-1999". Frontera Norte, 12(23): 87-117. 
Monárrez, J. E. 2002. "Feminicidio sexual serial en Ciudad Juárez: 1993-2001". Debate Feminista, 25: 279-305.

Monárrez, J. E. 2009. Trama de una injusticia. Feminicidio sexual sistémico en Ciudad Juárez. Tijuana: Colegio de la Frontera Norte.

Monárrez, J. E. 2012. "Violencia extrema y existencia precaria en Ciudad Juárez". Frontera Norte, 24(48): 191-199.

Munevar, D. I. 2012. "Delito de femicídio: muerte violenta de mujeres por razones de género". Revista Estudios Socio-Jurídicos, 14(1): 35-75.

Pérez, M. E. 2005. "Las Organizaciones No Gubernamentales en Ciudad Juárez y su lucha contra la violencia de género". Nóesis. Revista de Ciencias Sociales y Humanidades, 15(28): 147-167.

Pérez, M. E. y Padilla, H. 2002. "Interpretaciones locales sobre la violencia en contra de las mujeres en Ciudad Juárez". Revista de estudios de género. La ventana, 15: 195-230.

Pineda, S. y Herrera, L. A. 2007. "Ciudad juárez: Las sociedades de riesgo en la frontera norte de México." Fermentum: Revista Venezolana De Sociologia y Antropologia, 17(49): 419-433.

Radford, J. y Russell, D. E.H. 1992. Femicide: The Politics of Woman Killing. New York: Twayne Publishers.

Ravelo, P. y Sánchez, S. 2006. "Resistencia individual y colectiva ante la violencia de género. la experiencia de las obreras de las maquiladoras de Ciudad Juárez". Revista de estudios de género. La ventana, 24, 380-404.

Risse, T. y Kathryn K. 1999. "The Socialization of International Human Rights Norms into Domestic Practices: Introduction." En:T. Risse, S. C. Ropp y K. Sikkink (eds.), The power of human rights: international norms and domestic change. Nueva York: Cambridge University Press.

Rodríguez, R. 2008. "Del crimen pasional a la violencia de género: evolución y su tratamiento periodístico". Ámbitos, 17, 171-188.

Russell, D. E.H. 2006. "Definición de Feminicidio y Conceptos Relacionados". En: D. Russell y R. Harmes (Eds.), Feminicidio: una perspectiva global. México, D.F.: CEllCHUNAM.

Rusell, D. E.H. 2012. " «Femicide» - The Power of a Name". En C. Laurent, M. Platzer y M. Idomir (2013), Femicide A Global Issue that Demands Action. Vienna: Academic Council on the United Nations System (ACUNS) Vienna Liaison Office.

Sánchez, E. 2007. "Feminicidio y maquila en Ciudad Juárez". ICEV. Revista d'Estudis de la Violencia, 2, 1-12.

Vives-Cases, C., Torrubiano-Domínguez, J. y Álvarez-Dardet, C. 2008. “Distribución temporal de las denuncias y muertes por violencia de género en españa en el período 19982006". Revista Española de Salud Pública, 82(1): 91-100. 


\section{ARTICULOS/ARTICLES}

¿Cómo afrontar la obesidad en una sociedad digital? Respuestas desde el campo del Análisis Aplicado de la Conducta / How to treat obesity in a digital society? Answers from the Applied Behavior Analysis field

Ivan N. Martinez-Salazar y Erika Acevedo-Stefanoni

Femicidio y feminicidio: Un análisis de las aportaciones en clave iberoamericana / Femicide and Feminicidio: an analysis in Latinoamerican perspective

Santiago Boira, Chaime Marcuello-Servós, Laura Otero, Belén Sanz Barbero

y Carmen Vives-Cases

Grupos de ayuda mutua en personas con problemas severos de salud mental en el modelo de la recuperación. La inclusión del cuerpo / Self-help groups for people with severe mental health recovery model. Inclusion body

María José Rodríguez Sánchez, Matilde Blanco Venzalá y Clara Fernández Burraco

Págs 47-64

Cambio, desarrollo social y movimientos sociales en un contexto internacional en África

Subsahariana (Tanzania) / Change, social development and social movements in an international context in subsaharian Africa (Tanzania)

Roser Manzanera Ruiz

"Yo también quiero ser madre": Acceso a la maternidad en familias no heteronormativas /

"I want to be a mother too": Access to motherhood in non-heteronormative families

Gloria Álvarez-Bernardo y Nuria Romo-Avilés

Un aspecto de salud desapercibido y desatendido: los riesgos de caída domiciliaria en edad avanzada / An unnoticed and neglected health aspect: risks for domestic falls in older age José Azoh Barry

Teoría de la Impetración de la justicia. Por la necesaria ciudadanización de la justicia y la paz / Theory of supplication of Justice. For the necessary citizenization of the justice and peace

Francisco Javier Gorjon Gómez

Págs 113-131

\section{RESEIIAS/REVIEWS}

Antonio López Peláez (coord.). Social Work Challenges in the XXI Century: Perspectives from the USA / Retos para el Trabajo Social en el siglo XXI: perspectivas desde los Estados Unidos de América

(por Raquel Pérez García)

Págs 133-137

Enrique Pastor Seller y María Asunción Martínez Román (coords.). Trabajo Social en el siglo XXI. Una perspectiva internacional comparada / Social Work in the 21st century. An international comparative perspective (por María de las Olas Palma García) 